Check for updates

Cite this: RSC Adv., 2017, 7, 37241

Received 2nd June 2017

Accepted 22nd July 2017

DOI: $10.1039 / c 7 r a 06160 a$

rsc.li/rsc-advances

\section{Enhancements inside and outside the junctions of Ag colloidal dimers $\uparrow$}

\author{
Hyeokjin Yoon (D) and Jung Sang Suh (DD *
}

We measured experimentally the enhancements inside and outside the junctions of Ag colloidal dimers. We prepared two kinds of substrates for surface-enhanced Raman scattering (SERS) by using three- and fourstep immobilization methods. The three-step method consists of the following procedures: immobilization of Ag colloidal particles, adsorption of target molecules on the immobilized colloidal particles, and a second immobilization. For the four-step method, aniline is adsorbed in the second step, while target molecules are adsorbed in the fourth step. We optimized the diameter of Ag colloidal particles to achieve a high Raman enhancement from their dimers. When $\mathrm{Ag}$ sols, purified by centrifugation, were used in the immobilizations, dimers were predominantly formed with very few trimers formed. The substrate fabricated by the three-step method showed 6.5 times higher SERS intensity than the substrate fabricated by the four-step method. In both methods, the dimers were formed during the second immobilization. Therefore, the intensity difference was due to the fact that some target molecules were present at the junctions for the former substrate, while none at the junctions for the latter substrate. The enhancement on the dimers was about $1.03 \times 10^{8}$ for the substrate prepared by the three-step method, while $1.48 \times 10^{7}$ for the substrate prepared by the four-step method. The value of $1.48 \times 10^{7}$ corresponded to the enhancement outside the junctions, since no target molecules could be present at the junctions of the dimers for the substrate prepared by the four-step method. From the intensity difference between the two kinds of dimers, the percentage of the enhancement contributed by the target molecules presenting inside the junctions of the dimers where benzenethiol could not be adsorbed by diffusion was calculated to be about $86 \%$. We estimated the surface area inside the junctions where the sum of the SERS signals was corresponding to $86 \%$ of the total SERS signals by a finite difference time domain (FDTD) calculation. It was in the range of $0.5-0.7 \%$ of the total surface area. Based on this data, we could calculate roughly the enhancement inside the junctions. The enhancements inside and outside the junctions of the dimers were the order of $10^{10}$ and $10^{7}$, respectively.

\section{Introduction}

The Raman signals of molecules adsorbed on the surface of metal nanoparticles (NPs) like Ag and Au colloidal particles are enhanced hugely when their localized surface plasmon resonance (LSPR) is excited by irradiation with light. ${ }^{\mathbf{1 , 2}}$ This phenomenon is well-known as surface-enhanced Raman scattering (SERS). ${ }^{3}$ Recently, SERS has received much attention as a probing technique for biosensors because of its high sensitivity ${ }^{4-7}$ it is known that SERS can detect even single molecules. ${ }^{8-10}$ To achieve such high-sensitivity detection, a very high enhancement - up to $10^{11}$ for non-resonant molecules and $10^{8}$ for resonant molecules - may be required. ${ }^{11,12}$ The strongest signal enhancement in SERS substrates is obtained from so-called

Department of Chemistry, Seoul National University, Seoul 08826, Republic of Korea. E-mail: jssuh@snu.ac.kr; Fax:+82-2-875-6636; Tel: +82-2-880-7763

$\dagger$ Electronic supplementary information (ESI) available. See DOI: 10.1039/c7ra06160a "hotspots", which may be the junctions between two or more closely spaced metal NPs. ${ }^{13-21}$ A dimer of $\mathrm{Ag}$ and $\mathrm{Au}$ colloidal particles is undoubtedly one of the simplest junction systems.

In performing most calculations of the SERS enhancements, ${ }^{13,22,23}$ it was assumed that probe molecules adsorbed on the areas outside the hotspots do not contribute meaningfully to the SERS signals detected because the enhancement inside the hotspots is much higher than that outside the hotspots. However, the hotspot regions are relatively very narrow compared to the total surface area. Therefore, the number of probe molecules adsorbed on the areas outside the hotspots will be much larger than that adsorbed at the hotspots. Since Raman signals are proportional to the number of molecules, the SERS signals contributed by the molecules adsorbed on the areas outside the hotspots would not be so small as their contribution could be ignored. Therefore, it is necessary to distinguish the enhancements inside and outside the hotspots for a given junction system. In this study, we measured experimentally the enhancements inside and outside the junctions of Ag colloidal dimers. 
Lee et al. of our group developed, in 2011, a three-step immobilization method to fabricate the clusters of $\mathrm{Ag}$ colloidal particles. ${ }^{24}$ However, it was hard to control the cluster size. Recently, we improved the three-step immobilization method by increasing the surface charge of colloidal particles. ${ }^{25}$ When silver sols purified by centrifuging were used in the immobilizations, dimers were formed predominantly with forming very few trimers (see Scheme 1). Also, we optimized the diameter of Ag colloidal particles to accomplish a high Raman signal enhancement from their dimers. Also, we developed a four-step immobilization method: immobilization of $\mathrm{Ag}$ colloidal particles, and then adsorbing weakly adsorbed molecules like aniline instead of target molecules on the immobilized colloidal particles, and then immobilization again, and finally adsorbing target molecules. ${ }^{25}$ The substrate fabricated by the three-step method showed 6.5 times higher in SERS intensity than that of the substrate fabricated by the four-step method. This is due to the fact that some target molecules could present at the junctions of the dimers for the substrates prepared by the three-step method, while none at the junctions for the substrates prepared by the four-step method. In this

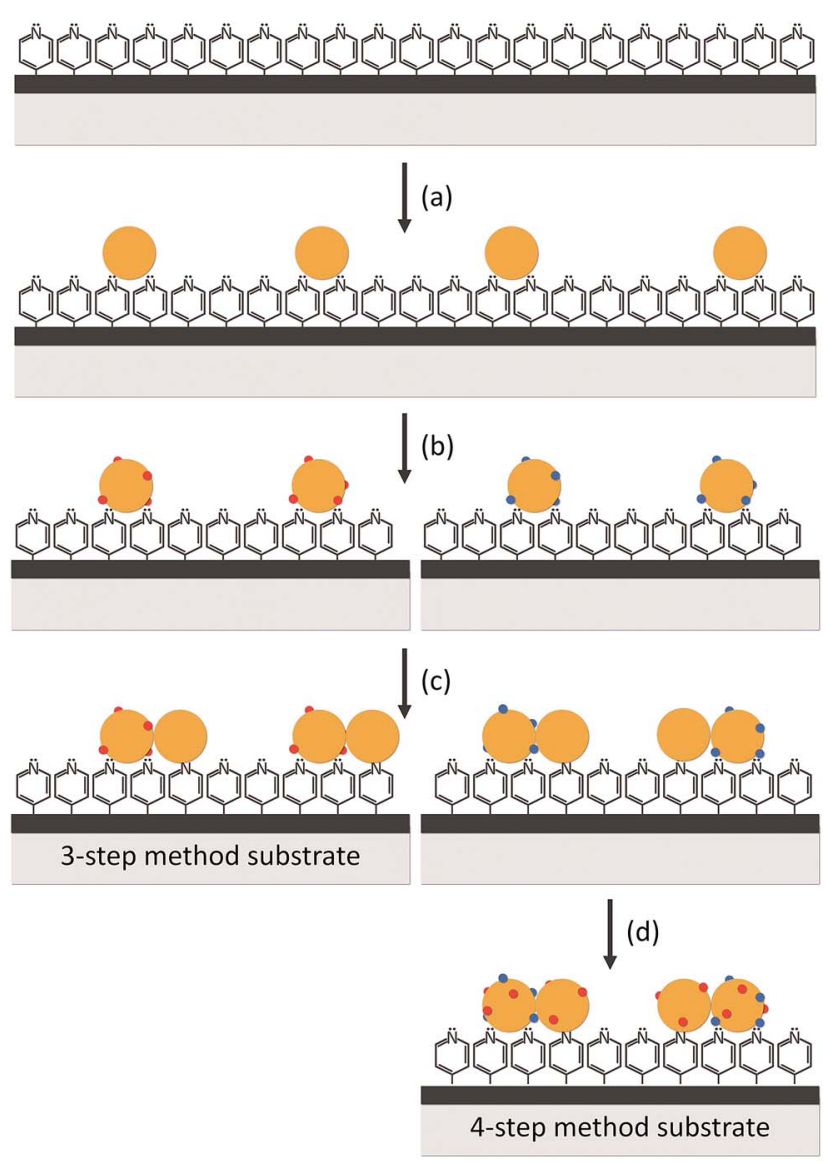

Scheme 1 A schematic of the fabrication of SERS substrates consisting of dimers of Ag or Au colloidal particles; (a) immobilization of the colloidal particles on a cover glass coated with P4VP, (b) adsorption of target molecules (in the left column) or aniline (in the right column), (c) second immobilization, and (d) adsorption of target molecules. study, from the SERS intensity difference we distinctly calculated the enhancements inside and outside the junctions of the dimers. They were the order of $10^{10}$ and $10^{7}$, respectively.

\section{Experimental section}

The diameter of Ag colloidal particles was controlled by using a seed-mediated process. ${ }^{26}$ The Ag seed solution was prepared by mixing $0.30 \mathrm{~mL}$ of $10 \mathrm{mM}$ silver nitrate solution and $20 \mathrm{~mL}$ of $1 \mathrm{mM}$ trisodium citrate solution and then rapidly injecting icecold $1.8 \mathrm{~mL}$ of $10 \mathrm{mM}$ sodium borohydride solution. The mixture was then stirred vigorously and then allowed to stand at room temperature for $3 \mathrm{~h}$. Silver sols whose colloidal particles were $28 \mathrm{~nm}$ in average diameter were synthesized as follows: 9 $\mathrm{mL}$ of the $\mathrm{Ag}$ seed solution, $11 \mathrm{~mL}$ of water, and $1.2 \mathrm{~mL}$ of $20 \mathrm{mM}$ sodium ascorbate solution were mixed together. To this solution, $1.2 \mathrm{~mL}$ of $10 \mathrm{mM}$ silver nitrate solution was injected rapidly and then the resulting mixture was stirred vigorously. The ions or molecules present in the silver sols were reduced by removing the supernatant with a pipette after centrifuging the silver sols at $13500 \mathrm{rpm}$ for $40 \mathrm{~min}$. The same volume of distilled-deionized water was added to the residue left behind and the resulting mixture was then shaken for $1 \mathrm{~min}$.

$0.3 \mathrm{~g}$ of poly(4-vinyl pyridine) (P4VP) was dissolved in ethanol $100 \mathrm{~mL}$. This polymer solution $(100 \mu \mathrm{L})$ was dropped on a cover glass $(2.2 \mathrm{~cm} \times 2.2 \mathrm{~cm})$ and then the glass plate was rotated at $3000 \mathrm{rpm}$ for $1 \mathrm{~min}$. The surface of the cover glass was then washed by dipping it in ethanol several times. The cover glass coated with P4VP was placed in a Petri dish containing $3.0 \mathrm{~mL}$ of silver sols to immobilize Ag colloidal particles on it. After immobilization, the surface was washed with water and then the cover glass was dried by blowing nitrogen gas. To adsorb benzenethiol or aniline on the immobilized Ag colloidal particles, the cover glass was dipped in a $3.0 \mathrm{~mL}$ ethanol solution of benzenethiol $(100 \mathrm{nM})$ or aniline $(200 \mathrm{nM})$ for $24 \mathrm{~h}$. After washing the surface with ethanol, the cover glass was again placed in the Petri dish containing the $3.0 \mathrm{~mL}$ colloidal solution of $\mathrm{Ag}$ for various durations to immobilize more colloidal particles. The surface of the cover glass (SERS substrate) was then washed with water and dried.

A micro-Raman system, which was equipped with a homemade sample stage, monochromator (SPEX $500 \mathrm{M}$ ), and CCD camera cooled with liquid nitrogen (Roger Scientific 7346-001 Model), was used to measure normal Raman and SERS spectra. Two different objective lenses whose NAs were 0.25 and 0.5 were used. Raman spectra were acquired at $514.5 \mathrm{~nm}$. The laser power incident on the sample was approximately $10 \mu \mathrm{W}$, and acquisition time of $1 \mathrm{~s}$ were used. The Raman spectrum of liquid benzenethiol was obtained from a sample contained in a capillary (364 $\mu \mathrm{m}$ in diameter). Raman frequencies were corrected by using the Raman peaks of a mixture of toluene and acetonitrile $(1: 1 \mathrm{v} / \mathrm{v})$. The UV-Vis extinction spectra of Ag colloidal particles immobilized on the cover glass coated with P4VP were obtained by a transmission method using the cover glass coated with P4VP as the reference material. The morphology of the particles was analyzed by using a highresolution transmission electron microscope (HR-TEM; JEOL, 
JEM-3000F (300 kV)) and scanning electron microscope (SEM; JEOL Ltd. JSM6700F (10 kV)). The FDTD method was used to calculate the local electric fields (Lumerical Solutions, Inc.).

\section{Results and discussion}

Fig. 1 shows the SEM image of the substrate after the first immobilization (a), and SEM images of two different SERS substrates prepared by the three-step (b) and four-step (c) immobilization methods [the magnified SEM image of Fig. 1b was shown in Fig. $\mathrm{S} 1 \dagger]$. The average diameter of the colloidal particles was about $28 \mathrm{~nm}$, which showed the highest SERS intensity from their dimers (see Fig. S2 and S3†). In the threestep method, we immobilized Ag colloidal particles on a cover glass coated with P4VP, and then adsorbed benzenethiol as a target molecule on the immobilized particles in the first step, and finally immobilized Ag colloidal particles again. In the fourstep method, we immobilized Ag colloidal particles, and then adsorbed aniline instead of benzenethiol on the immobilized particles, and then immobilized Ag colloidal particles again, and finally adsorbed benzenethiol. When target molecules or aniline were adsorbed on the surface of Ag colloidal particles immobilized, the surface charge of the $\mathrm{Ag}$ colloidal particles immobilized reduced, and in the second immobilization the repulsion force between the $\mathrm{Ag}$ particles immobilized and fresh $\mathrm{Ag}$ colloidal particles approaching to them decreased, and the dimerization could take place. In both methods, the first and second immobilization times were the same as 10 and $30 \mathrm{~min}$, respectively. The adsorption times of aniline and benzenethiol molecules were all the same as $24 \mathrm{~h}$. In addition, the total amount of benzenethiol adsorbed on both the SERS substrates fabricated on a cover glass $(22 \mathrm{~mm} \times 22 \mathrm{~mm})$ was the same $(3.0$ $\left.\times 10^{-10} \mathrm{~mol}\right)$. In the immobilizations, we used silver sols purified by centrifuging. The purification of silver sols reduced the concentration of the ions present in the sols significantly. Consequently, the net surface charge of the Ag colloidal particles increased and the force of repulsion between the colloidal particles increased significantly. All the colloidal particles were immobilized individually, without forming any dimers in the first immobilization (see Fig. 1a). This is due to the increased surface charge of the purified Ag colloidal particles. In the second immobilization, the dimers of $\mathrm{Ag}$ colloidal particles were formed dominantly, while very few trimers were formed (see Fig. 1b and c) [in the SEM image (a), there are 117 monomers. In the image (b), there are 75 monomers, 72 dimers and 2 trimers. In the SEM image (c), 74 monomers, 77 dimers, and 2 trimers]. It should be mentioned that when pristine silver sols were used in the immobilizations, not all the colloidal particles were immobilized individually during the first immobilization and relatively large clusters such as trimers and tetramers were also formed along with the dimers during the second immobilization (see Fig. $\mathrm{S} 4 \dagger$ ).

Fig. 2 shows the extinction and SERS spectra measured from the two substrates whose SEM images are shown in Fig. 1b and c. The black spectra were measured from the substrate prepared by the three-step immobilization method, while the red ones from the substrate prepared by the four-step method. Both
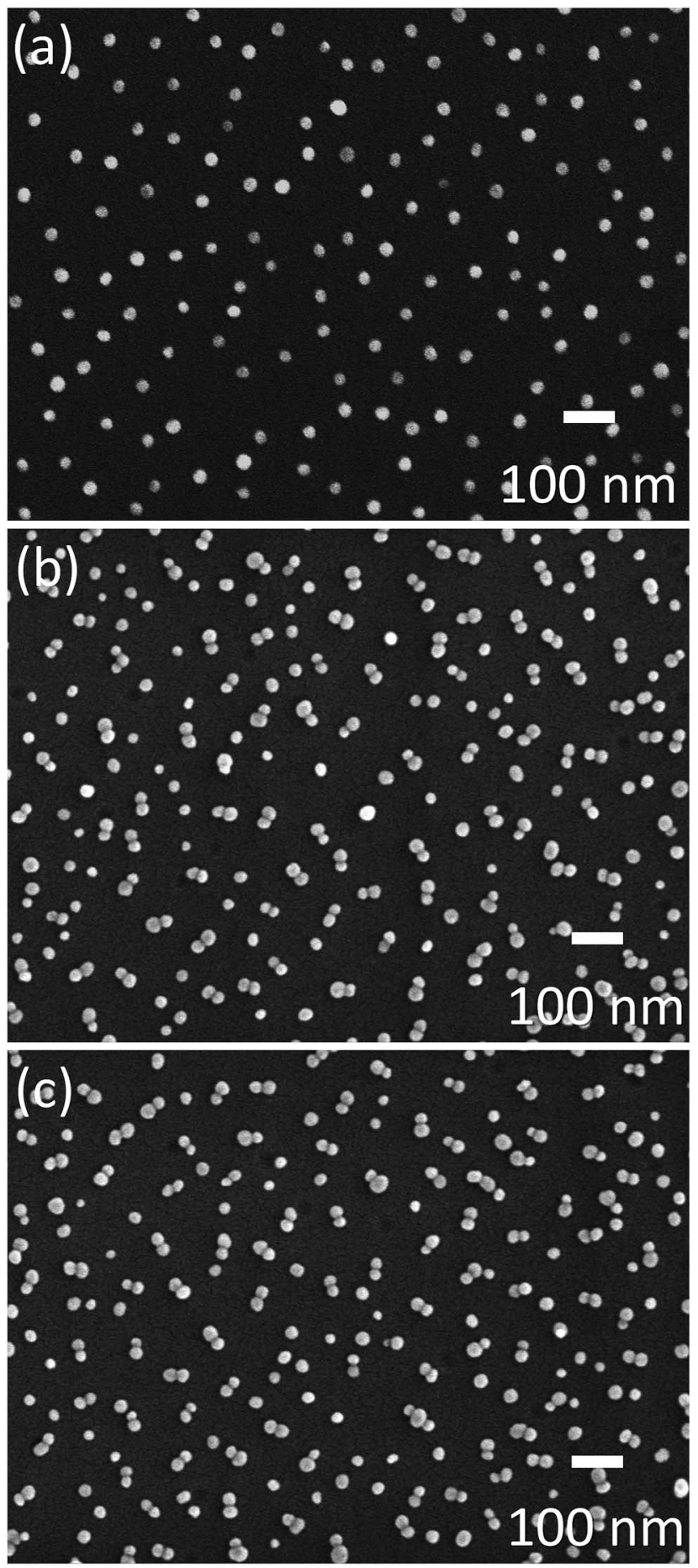

Fig. 1 SEM image of the substrate after the first immobilization (a), and SEM images of two different SERS substrates prepared by the threestep (b) and four-step (c) immobilization methods. In both methods, the first and second immobilization times were the same as 10 and 30 min, respectively. The total amount of benzenethiol adsorbed on each substrate was the same. In the SEM image (a); there are 117 monomers. In the image (b); 75 monomers, 72 dimers, and 2 trimers. In the SEM image (c); 74 monomers, 77 dimers, and 2 trimers.

extinction spectra were very similar in terms of the spectral shape and intensity. There are two LSPR peaks at 380 and $519 \mathrm{~nm}$. The former peak is attributed to the transverse mode of 

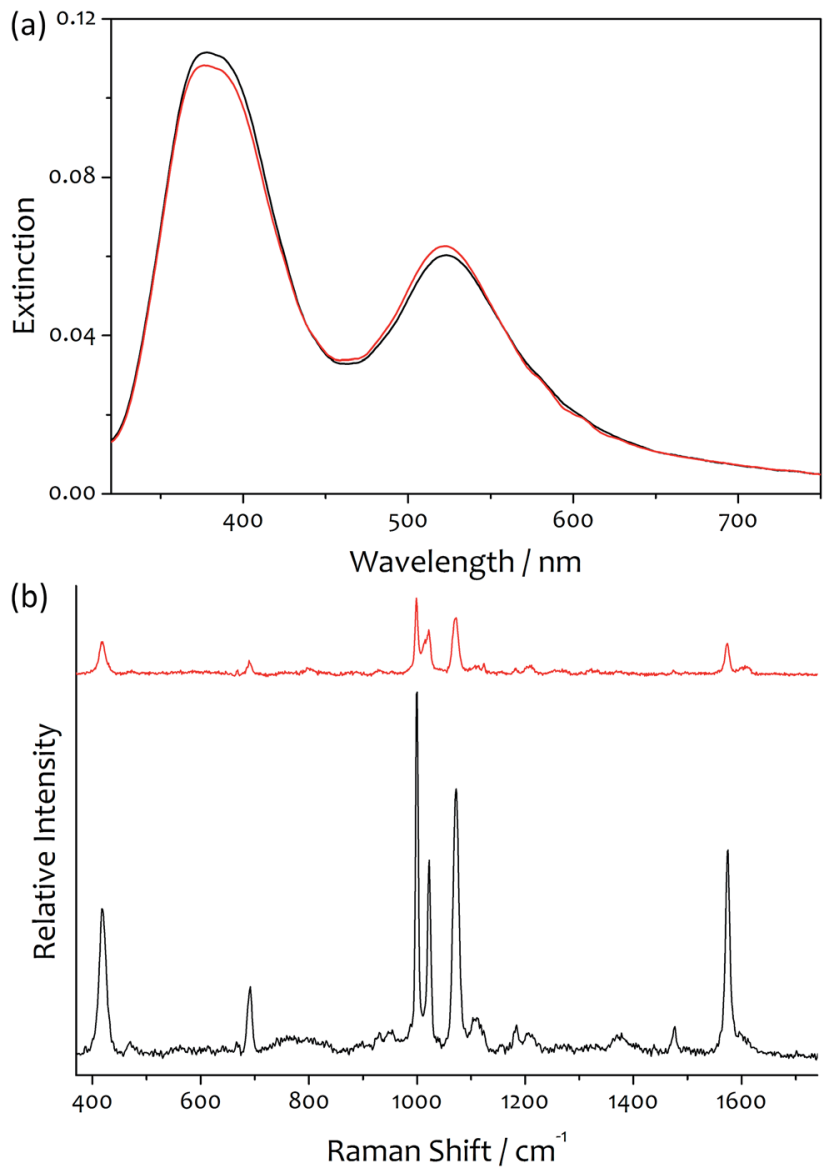

Fig. 2 Comparison of (a) UV-Vis extinction and (b) SERS spectra measured from the two different substrates whose SEM images are shown in Fig. 1. The black spectra were obtained from the substrate prepared by the three-step method, while the red spectra from the substrate prepared by the four-step method. The total amount of benzenethiol adsorbed on each substrate was the same. The intensity of the black SERS spectrum was approximately 6.5 times stronger than that of the red SERS spectrum. The acquisition time was $1 \mathrm{~s}$ and the $50 \times$ objective lens was used. For two substrates, the total number of benzenethiol molecules adsorbed was the same and the adsorption time of benzenethiol was also the same as $24 \mathrm{~h}$.

the dimers of Ag colloidal particles, while the latter is attributed to the longitudinal mode. Ag colloidal particles immobilized in isolation showed a single LSPR peak at $390 \mathrm{~nm}$ (see Fig. S4†).

The SERS spectra measured from the two substrates are very strong and well characterized. The SERS peaks observed from the SERS spectra correspond to the modes of the benzene ring of benzenethiol. ${ }^{27}$ For example, the peak at $1573 \mathrm{~cm}^{-1}$ corresponds to $\nu_{8 \mathrm{a}}$, while that at $1000 \mathrm{~cm}^{-1}$ corresponds to $\nu_{12}$. The peak at $417 \mathrm{~cm}^{-1}$ corresponds to $\nu_{7 \mathrm{a}}$ and contributions from the C-S stretching vibration $\left(\nu_{\mathrm{CS}}\right)$. It should be noted that no peaks corresponding to P4VP and aniline were observed. When a $50 \times$ objective lens, whose laser beam spot size was $\sim 1 \mu \mathrm{m}$ in diameter, was used, the standard deviation of relative intensities measured at 10 different points of each sample was less than 5\% (see Fig. S5 and Table S1 $\dagger$ ). It should be mentioned that the SERS intensity was just slightly fluctuated, randomly, during measurements of a series of SERS spectra (see Fig. S6†). This may mean that no laser induced thermal desorption or surface diffusion of benzenethiol molecules took place during measurements. This is due to the fact that benzenethiol is strongly chemisorbed on the silver surface.

When the $\lambda_{\max }$ of the longitudinal mode of $\mathrm{Ag}$ dimers was around $519 \mathrm{~nm}$, the highest SERS intensity was obtained by excitation with a $514.5 \mathrm{~nm}$ laser line. The optimum value of the $\lambda_{\max }$ was somewhat greater than the wavelength of the excitation light. This is owing to the fact that the SERS intensity is strongly correlated to the extinction at excitation and Stokes wavelengths. ${ }^{28}$ When a $514.5 \mathrm{~nm}$ laser line is used for Raman excitation, the Stokes Raman scattering wavelength becomes greater than the laser line wavelength by 10-45 $\mathrm{nm}$ depending on the modes. Therefore, the SERS intensity reaches the maximum value when the $\lambda_{\max }$ is slightly greater than the wavelength of the excitation light. A $\lambda_{\max }$ of $519 \mathrm{~nm}$ was achieved when dimers were formed from $28 \mathrm{~nm}$ (average diameter) Ag colloidal particles.

Although their extinction spectra were very similar in terms of the spectral shape and intensity, the SERS intensity measured from the substrate prepared by the three-step method was approximately 6.5 times higher than that measured from the substrate prepared by the four-step method. The LSPR of the monomers of Ag colloidal particles occurs around $390 \mathrm{~nm}$, which is too far to be excited by a $514.5 \mathrm{~nm}$ light. Therefore, the SERS signal in these substrates was mainly contributed by the molecules adsorbed on the dimers. Since the transverse mode of the dimers of Ag colloidal particles occurs at $380 \mathrm{~nm}$, which is also too far to be excited by a $514.5 \mathrm{~nm}$ light. Therefore, the SERS signals measured by excitation with a $514.5 \mathrm{~nm}$ light were mainly contributed by the longitudinal mode of the dimers [it should be noted that we ignored the contribution of the trimers to the observed SERS signals because the number of the trimers was very few and their longitudinal mode was somewhat apart from the laser line used]. In the three-step method, benzenethiol served as the target molecule and was adsorbed on the Ag colloidal particles immobilized during the first immobilization process. There are 117 individually immobilized Ag colloid particles in the SEM image of Fig. 1a, and 72 dimers in the SEM image of Fig. 1b. Therefore, the percentage of dimerization among the $\mathrm{Ag}$ colloidal particles immobilized in the first immobilization was about $62 \%[=100 \% \times 72 / 117]$. This means that about $62 \%$ of benzenethiol molecules were adsorbed on the dimers [it should be mentioned that some colloidal particles were adsorbed individually even during the second immobilization]. In the four-step method, benzenethiol was adsorbed on the surface colloidal particles immobilized on the substrate, the percentage of the colloidal particles composing the dimers was found to be about $66 \%[=100 \% \times 77 \times 2 /(74 \times 1+77 \times 2+2 \times$ 3)] (see Fig. 1c). This means that about $66 \%$ of the benzenethiol molecules were adsorbed on the dimers of the substrate prepared by the four-step method. For the substrate prepared by the three-step method, $62 \%$ of target molecules were adsorbed on 72 dimers, while for the substrate prepared by the four-step method $66 \%$ of target molecules on 77 dimers. Therefore, each dimer of two substrates had almost the same number of target 
molecules on its surface $[62 \% / 72 \approx 66 \% / 77 \approx 0.86 \%]$. Therefore, it can be concluded that the number of benzenethiol molecules adsorbed on each dimer was almost the same in both the substrates.

In the three-step method, benzenethiol was adsorbed on the $\mathrm{Ag}$ colloidal particles immobilized during the first immobilization process, and the dimers were formed in the second immobilization process. Therefore, a certain portion of benzenethiol molecules would present at the junctions of the dimers. In the four-step method, benzenethiol was adsorbed on the surface of all the colloidal particles present on the substrate. However, in order to be adsorbed at the junctions of the already formed dimers, the benzenethiol molecules needed to penetrate into the narrow gap of the junctions between two attached colloidal particles. During the course of diffusion to reach the junctions, the benzenethiol molecules might have collided many times with the surface of the Ag colloidal particles composing the dimers. Since benzenethiol was strongly chemisorbed, it is likely that it was adsorbed on the surface after a few collisions before reaching to the junctions. This means that in the four-step method the benzenethiol molecules could not be adsorbed at the junctions. It should be noted that the spreading of benzenethiol molecules on the surface of the dimers was not the same in the two samples. For the substrates prepared by the three-step method, benzenethiol was adsorbed on the surface of the colloidal particles immobilized in isolation and then the dimers were formed by the attachment of fresh colloidal particles to this assembly. However, for the substrates prepared by the four-step method benzenethiol was adsorbed on the already formed dimers. Therefore, in the substrates formed by the three-step method, benzenethiol was adsorbed on only one particle composing the dimers, while in the substrates formed by the four-step method, it was adsorbed on the both particles composing the dimers. Nevertheless, the SERS intensity would not be affected significantly by this difference. This is because each colloidal particle composing the dimers in both the substrates may show a similar SERS effect. Therefore, it can be concluded that the difference in the SERS intensity of the two kinds of substrates (Fig. 2) resulted from the fact that some benzenethiol molecules existed at the junction regions in the substrates prepared by the three-step method unlike the substrates prepared by the four-step method.

The SERS enhancement was calculated by comparing the intensity of the $1575 \mathrm{~cm}^{-1}$ peak in the SERS spectrum with that in the normal Raman spectrum. In calculation of the number of molecules adsorbed on the surface of $\mathrm{Ag}$ colloidal particles, we assumed that all the molecules contained in a $3.0 \mathrm{~mL}$ ethanol solution of $100 \mathrm{nM}$ benzenethiol were completely adsorbed on the surface of the colloidal particles immobilized on the cover glass $(22 \mathrm{~mm} \times 22 \mathrm{~mm})$. A complete adsorption might be reasonable, since benzenethiol was strongly chemisorbed on silver surface, the adsorption time was long enough as $24 \mathrm{~h}$, and the surface coverage was well below a monolayer. By a simple calculation, the amount of the molecules contained in the 3.0 $\mathrm{mL}$ benzenethiol solution was corresponded to about $3.6 \%$ of the surface atoms of Ag colloidal particles immobilized during the first immobilization. The average enhancement factor was calculated to be approximately $6.4 \times 10^{7}$ for the SERS substrate prepared by the three-step method (see Fig. S7† and the calculation), while $9.8 \times 10^{6}$ for the SERS substrate prepared by the four-step method (see Fig. S8 $\dagger$ and the calculation).

The molecules adsorbed on the isolated Ag colloidal particles could not contribute to the calculated enhancement, since the LSPR of them is near $390 \mathrm{~nm}$, which is too far to be excited by a $514.5 \mathrm{~nm}$ light. Therefore, the actual enhancement on the dimers might be much higher than the calculated value. About 62 and $66 \%$ of the benzenethiol molecules were adsorbed on the dimers of the substrates prepared by the three- and fourstep methods, respectively. If we count this factor, the enhancement on the dimers becomes about $1.03 \times 10^{8}[=6.4 \times$ $\left.10^{7} / 0.62\right]$ for the substrate prepared by the three-step method. For the substrate prepared by the four-step method, it becomes about $1.48 \times 10^{7}\left[=9.8 \times 10^{6} / 0.66\right]$. The value of $1.48 \times 10^{7}$ corresponds to the enhancement outside the junctions, since no target molecules could present at the junctions of the dimers for the substrate prepared by the four-step method.

The enhancement measured from the substrate prepared by the three-step method was approximately $7.0\left[=1.03 \times 10^{8} / 1.48\right.$ $\left.\times 10^{7}\right]$ times stronger than that measured from the substrate prepared by the four-step method. As discussed in above, a certain portion of benzenethiol molecules would present inside the junctions of the dimers for the substrate prepared by the three-step method, while none inside the junctions for the substrate prepared by the four-step method. Therefore, the enhancement difference of 7.0 times is surely due to the contribution by the target molecules located inside the junctions. The percentage of the enhancement contributed by them is calculated to be about $86 \%[=(1-1 / 7.0) \times 100 \%]$. To calculate the enhancement inside the junctions, which act as the hotspots, we have to know the amount of benzenethiol adsorbed on the surface area inside the junction regions. This amount could be estimated roughly if we know the surface area inside the junction regions where benzenethiol could not be adsorbed by diffusion. Unfortunately, we could not find any proper experiment methods to measure the surface area or theory to estimate it. However, the value of $86 \%$ could give us a clue to estimate the surface area inside the junction regions where benzenethiol could not be adsorbed by diffusion. To estimate the surface area, we did a FDTD calculation (Fig. S9†). The percent of the SERS intensity contributed by the molecules adsorbed on the spherical cap area of the model shown in Fig. 3 was calculated by varying the height of the spherical cap $(h)$ and the gap between two colloidal particles $(g)$. Where $r$ is the radius of the colloidal spheres, and it is $14 \mathrm{~nm}$ for the colloidal particles used. The calculated data were summarized in Table S2. $\dagger$ $86 \%$ of the total SERS intensity is obtained from the spherical cap area when $g=0.1 \mathrm{~nm}$ and $h=0.019 \mathrm{~nm}$, or $g=0.5 \mathrm{~nm}$ and $h$ $=0.15 \mathrm{~nm}$, or $g=0.7 \mathrm{~nm}$ and $h=0.20 \mathrm{~nm}$. The minimum distance between edges of two symmetrical spherical caps $(l ; l=$ $g+2 h$ ) is $0.14,0.8$ and $1.1 \mathrm{~nm}$, respectively. We assumed that target molecules could not be adsorbed on the surface of the spherical caps by diffusion. The molecular size of benzenethiol in the long axis is about $0.7 \mathrm{~nm}$. If we consider the molecular size, for the case of $l=0.14 \mathrm{~nm}$ it is too narrow to be diffused by 


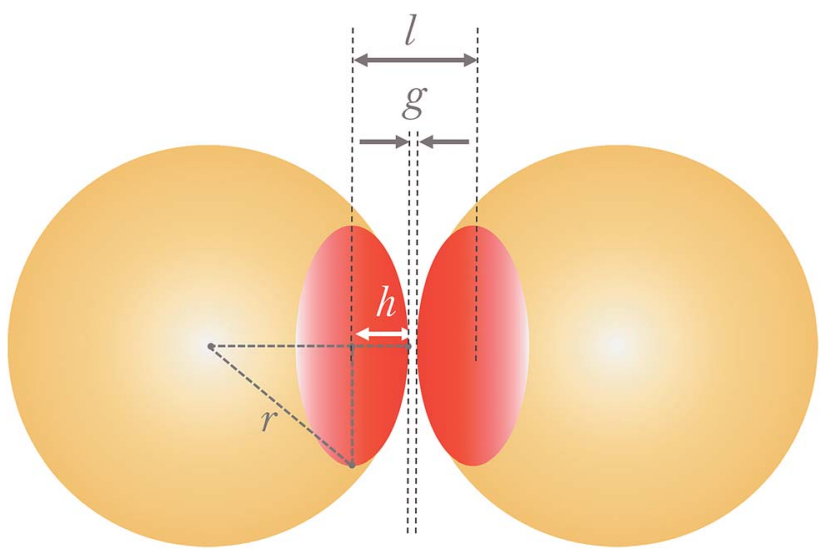

Fig. 3 A schematic showing our approach to estimate the number of target molecules adsorbed on the surface of the junction of a dimer The junction area is assumed to comprise a cap on the surface of each colloidal particle in the interparticle region of the dimer (red color).

the benzenethiol molecules. We believe that $l=0.8 \mathrm{~nm}$ or $1.1 \mathrm{~nm}$ is reasonable in this aspect. The surface of the spherical cap is given as $2 \pi r h .^{29}$ Therefore, the percent of the junction area is calculated as $2 \pi r h / 4 \pi r^{2}$. The percent is about 0.54 and $0.71 \%$ when $h=0.15$ and $0.20 \mathrm{~nm}$, respectively. Since the number of target molecules adsorbed on the surface inside the junction regions is proportional to its surface area, the enhancement inside the junction regions is estimated at about $1.6 \times 10^{10}\left[=1.0 \times 10^{8} \times 86 \% / 0.54 \%\right]$. The enhancement is 1.2 $\times 10^{10}$ when the percent of the junction area is $0.71 \%$. Therefore, it is concluded that the enhancement inside the junction regions where benzenethiol could not be adsorbed by diffusion is the order of $10^{10}$. The simulated Raman enhancement $\left(|E|^{4}\right)$ inside the junction regions is $4.4 \times 10^{8}$ and $6.0 \times 10^{7}$ when $h=$ 0.15 with $g=0.5 \mathrm{~nm}$ and $h=0.20 \mathrm{~nm}$ with $g=0.7 \mathrm{~nm}$, respectively. These values are much lower than those calculated based the percent of the junction area where benzenethiol could not be adsorbed by diffusion. The simulated enhancement is critically affected by the gap size between two colloidal particles of the dimers. However, it was very hard to measure the gap size from the SEM images. For an accurate inside enhancement of the junctions, we need an accurate method to figure out the surface area inside the junction regions where benzenethiol could not be adsorbed by diffusion.

\section{Conclusions}

We prepared two kinds of SERS substrates by using the three- and four-step immobilization methods. By using purified $\mathrm{Ag}$ sols in the immobilizations, we could fabricate the dimers of $\mathrm{Ag}$ colloidal particles predominantly with forming very few trimers. The net surface charge of the colloidal particles was increased by purification, and the force of repulsion between the colloidal particles was increased significantly. Consequently, the dimers formed from the purified colloidal particles might have surface charge densities high enough prevent the attachment of new purified colloidal particles to them, and very few trimers were formed. Also, we optimized the diameter of Ag colloidal particles so that the dimers had a high Raman signal enhancement. The optimum diameter was $28 \mathrm{~nm}$. Both substrates gave good SERS spectra. However, the spectral intensity measured from the substrate prepared by the three-step method was about 6.5 times higher than that measured from the substrate prepared by the four-step method. This was due to the fact that some of the target molecules could be present inside the junction regions for the substrate prepared by the three-step method, while none inside the junction regions for the one prepared by the four-step method. From the SERS intensity difference, we could distinctly calculate the enhancement factors inside and outside the junction regions of the dimers of Ag colloidal particles. It was found to be roughly the order of $10^{10}$ inside the junctions, while the order of $10^{7}$ outside the junctions.

\section{Acknowledgements}

This work was supported by Basic Study program through the National Research Foundation of Korea funded by the Ministry of Education, Science and Technology (2015R1D1A1A09058247), and the BK21 program.

\section{References}

1 K. L. Kelly, E. Coronado, L. L. Zhao and G. C. Schatz, J. Phys. Chem. B, 2003, 107, 668-677.

2 S. Link and M. A. El-Sayed, J. Phys. Chem. B, 1999, 103, 84108426.

3 B. Sharma, R. R. Frontiera, A.-I. Henry, E. Ringe and R. P. Van Duyne, Mater. Today, 2012, 15, 16-25.

4 K. W. Kho, U. S. Dinish, A. Kumar and M. Olivo, ACS Nano, 2012, 6, 4892-4902.

5 S. S. Sinha, S. Jones, A. Pramanik and P. C. Ray, Acc. Chem. Res., 2016, 49, 2725-2735.

6 G. Das, N. Patra, A. Gopalakrishnan, R. P. Zaccaria, A. Toma, S. Thorat, E. D. Fabrizio, A. Diaspro and M. Salerno, Analyst, 2012, 137, 1785-1792.

7 S. C. Vijayakumar, K. Venkatakrishnan and B. Tan, ACS Appl. Mater. Interfaces, 2017, 9, 5077-5091.

8 S. Nie and S. R. Emory, Science, 1997, 275, 1102-1106.

9 H. Xu, E. J. Bjerneld, M. Käll and L. Börjesson, Phys. Rev. Lett., 1999, 83, 4357-4360.

10 D.-K. Lim, K.-S. Jeon, H. M. Kim, J.-M. Nam and Y. D. Suh, Nat. Mater., 2010, 9, 60-67.

11 P. G. Etchegoin and E. C. Le Ru, Phys. Chem. Chem. Phys., 2008, 10, 6079-6089.

12 G. H. Gu and J. S. Suh, J. Phys. Chem. A, 2009, 113, 8529-8532.

13 E. C. Le Ru, E. Blackie, M. Meyer and P. G. Etchegoin, J. Phys. Chem. C, 2007, 111, 13794-13803.

14 G. H. Gu and J. S. Suh, Langmuir, 2008, 24, 8934-8938.

15 G. H. Gu and J. S. Suh, J. Phys. Chem. C, 2010, 114, 72587262.

16 S. J. Lee, A. R. Morrill and M. Moskovits, J. Am. Chem. Soc., 2006, 128, 2200-2201.

17 J. P. Camden, J. A. Dieringer, Y. Wang, D. J. Masiello, L. D. Marks, G. C. Schatz and R. P. Van Duyne, J. Am. Chem. Soc., 2008, 130, 12616-12617. 
18 J. A. Dieringer, R. B. Lettan II, K. A. Scheidt and R. P. Van Duyne, J. Am. Chem. Soc., 2007, 129, 16249-16256.

19 M. Rycenga, X. Xia, C. H. Moran, F. Zhou, D. Qin, Z.-Y. Li and Y. Xia, Angew. Chem., 2011, 123, 5587-5591.

20 P. H. C. Camargo, M. Rycenga, L. Au and Y. Xia, Angew. Chem., Int. Ed., 2009, 48, 2180-2184.

21 A. Hakonen, M. Svedendahl, R. Ogier, Z.-J. Yang, K. Lodewijks, R. Verre, T. Shegai, P. O. Andersson and M. Käll, Nanoscale, 2015, 7, 9405-9410.

22 M. V. Cañ amares, J. V. Garcia-Ramos, S. Sanchez-Cortes, M. Castillejo and M. Oujja, J. Colloid Interface Sci., 2008, 326, 103-109.

23 E. C. Le Ru, P. G. Etchegoin and M. Meyer, J. Chem. Phys., 2006, 125, 204701.
24 S. Lee, G. H. Gu and J. S. Suh, Chem. Phys. Lett., 2011, 511, 121-125.

25 H. Yoon and J. S. Suh, RSC Adv., 2017, 7, 28573-28579.

26 D. Steinigeweg and S. Schlücker, Chem. Commun., 2012, 48, 8682-8684.

27 M. Osawa, N. Matsuda, K. Yoshii and I. Uchida, J. Phys. Chem., 1994, 98, 12702-12707.

28 N. Félidj, J. Aubard, G. Lévi, J. R. Krenn, A. Hohenau, G. Schider, A. Leitner and F. R. Aussenegg, Appl. Phys. Lett., 2003, 82, 3095-3097.

29 CRC Standard Mathematical Tables, ed. W. H. Beyer, CRC Press, Boca Raton, FL, 28th edn, 1987, p. 130. 